\title{
Outage Performance of Double Stratospheric Platforms Diversity Systems
}

\author{
Georgios A. Karagiannis, Athanasios D. Panagopoulos \\ and John D. Kanellopoulos \\ School of Electrical and Computer Engineering \\ National Technical University of Athens \\ Athens, Greece \\ E-mail: georgios_karagiannis@ hotmail.com, \\ thpanag@ece.ntua.gr, ikanell@ece.ntua.gr
}

\author{
Miltiades C. Filippou \\ Mobile Communications Department \\ EURECOM Institute \\ Sophia Antipolis, France \\ E-mail: filippou@eurecom.fr
}

\begin{abstract}
High Altitude Platform Networks (HAPNs) comprise an emerging communication solution promising to exploit many of the best aspects of terrestrial and satellite-based systems. Nevertheless, while offering advantageous propagation characteristics, HAP networks are still subject to large fades due to atmospheric precipitation and especially due to rain, for the allocated high frequency bands, necessary though to deliver high data rates. Multi-HAP or orbital HAP diversity is an effective technique to reduce the large fade margins required to assure the specified quality of service $(\mathrm{QoS})$. Received signals from multiple HAPs can be combined at the terminal station with the use of various signal processing techniques. The subject of this paper is to present physical statistical models for the evaluation of the outage probability of a dual-HAP orbital diversity system using either SC (selection combining) or MRC (maximal ratio combining) techniques. The analytical probabilistic models are interesting since we have rain attenuation correlated fading channels. Extended numerical results are presented in the final section of the paper comparing MRC and SC reception techniques. Some significant conclusions are drawn.
\end{abstract}

Index Terms- High Altitude Platform Network (HAPN); orbital diversity; rain attenuation; log-normal distribution; maximal ratio combining; selection combining ;orbital diversity.

\section{INTRODUCTION}

High altitude/stratospheric platforms (HAPs) are either airships or planes that operate in the stratosphere, $17-22 \mathrm{~km}$ above the ground [1]. Such platforms have the potential capability of being deployed rapidly, using considerably less communications infrastructure than that required if delivered by a terrestrial network. Very recently, ITU-R has allocated several frequency bands for communication services for HAP networks, $\mathrm{L}$ and $\mathrm{S}$ bands are allocated for mobile communication services and for fixed communication services, at $47 / 49 \mathrm{GHz}$, and at $28 / 31 \mathrm{GHz}$ [2] .

At millimeter wave bands, the dominant fading mechanism is rain attenuation [2]. Many fade mitigation techniques have been proposed to increase the availability time of the millimeter wave radio systems [2] but the most efficient are the path diversity techniques. Their main drawbacks are that they are cost ineffective and need double capacity for their implementation. Nevertheless, in this paper, we examine a double diversiy scheme of a HAP Network (HAPN) from a propagation point of view. At the ground station we investigate the performance of either the Selection
Combining (SC) reception technique or the Maximal Ratio Combining (MRC) reception technique. In [4], the error performance of a selection combining site divesity configuration has been investigated. In this paper, an accurate propagation prediction model is presented for the outage probability taking into account the convective raincell model. Including the nonraining time, the lognormal distribution is adopted for the distribution of the unconditional point rainfall rate and the induced rain attenuation on the HAP links similarly with [4]. The bivariate lognormal distribution, is also assumed for the rain induced attenuation on the the two HAP link configuration. The correlation coefficient is calculated through a well-behaved physical model [5].

Finally, we present extented numerical results comparing the two techniques and investigate the impact of the system geometry on the outage probability.

\section{CALCULATION OF THE OUTAGE PROBABILITIES}

\section{A. Geometrical Calculations}

The system consists of two HAPs as shown in Fig.1 located at points A and B. These points are located at a height $\mathrm{H}$ above the Earth surface and their terrestrial projections' distance is $\mathrm{s}$ (length of the arc $A^{\prime} B^{\prime}$ ). The user is located at a distance $\mathrm{d}$ from the center $\mathrm{O}$ of the projections and at an angle $\omega$ which lays on the tangent surface of the point $O$.

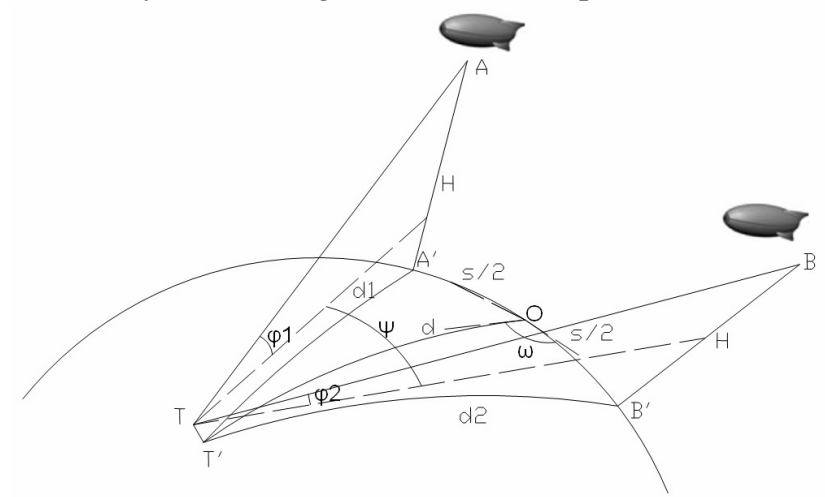

Figure 1. Double HAP System's geometry

From the spherical triangles OT'B' and OT'A' we can evaluate the distances $\mathrm{d} 1$ and $\mathrm{d} 2$ : 


$$
\begin{aligned}
d_{1}= & R_{e} \cdot \arccos \left[\cos \left(\frac{d}{R_{e}}\right) \cos \left(\frac{s}{2 R_{e}}\right)+\right. \\
& \left.+\sin \left(\frac{d}{R_{e}}\right) \sin \left(\frac{s}{2 R_{e}}\right) \cos (\pi-\omega)\right] \\
d_{2}= & R_{e} \cdot \arccos \left[\cos \left(\frac{d}{R_{e}}\right) \cos \left(\frac{s}{2 R_{e}}\right)+\right. \\
& \left.+\sin \left(\frac{d}{R_{e}}\right) \sin \left(\frac{s}{2 R_{e}}\right) \cos \omega\right]
\end{aligned}
$$

From the spherical triangle TA'B' we can evaluate the angle $\Psi$ formed by the projections of the radiopaths on the horizontal level at the user's location.

$$
\Psi=\arccos \left(\frac{\cos \left(\frac{s}{R_{e}}\right)-\cos \left(\frac{d_{1}}{R_{e}}\right) \cos \left(\frac{d_{2}}{R_{e}}\right)}{\sin \left(\frac{d_{1}}{R_{e}}\right) \sin \left(\frac{d_{2}}{R_{e}}\right)}\right)
$$

Given the height $\mathrm{H}$ of the HAPs and $\mathrm{h}$ of the user and having calculated the distances $d_{1}, d_{2}$ of the radiopaths' projections, we can evaluate the lengths $L_{1}, L_{2}$ of the radiopaths and the elevation angles $\phi_{1}, \phi_{2}$ of the radiopaths relative to the horizontal level.

$$
\begin{aligned}
& L_{i}^{2}=\left(R_{e}+h\right)^{2}+\left(R_{e}+H\right)^{2}- \\
& -2\left(R_{e}+h\right)\left(R_{e}+H\right) \cos \left(\frac{d_{i}}{R_{e}}\right), \quad(i=1,2)
\end{aligned}
$$

and

$$
\phi_{i}=\arcsin \left[\frac{\left(R_{e}+H\right) \cdot \cos \left(\frac{d_{i}}{R_{e}}\right)-\left(R_{e}+h\right)}{L_{i}}\right],(i=1,2)
$$

The rainfall height $\mathrm{H}_{\mathrm{r}}$ is taken from the ITU-R P.839 Recommendation [6]. Finally the effective radiopath lengths $L_{s_{1}}, L_{s_{2}}$ are given:

$$
L_{s_{i}}=\left\{\begin{array}{l}
{\left[(R e+H r)^{2}-(R e+h)^{2} \cos \phi_{i}^{2}\right]^{1 / 2}} \\
-(R e+h)^{2} \sin \phi_{i}, \phi_{i} \leqslant 10^{\circ} \\
\frac{H_{r}-h}{\sin \phi_{i}}, \phi_{i}>10^{\circ}
\end{array},(i=1,2)\right.
$$

\section{B. General Considerations}

The calculation of the outage probability of a specific link $i$ is directly related to the probability that the attenuation due to rain exceeds a certain value.

It is well known that the signal to noise ratio of a link under rain fading conditions is given [3]:

$$
S N R_{i}=S N R_{c s, i}-A_{i}(\mathrm{~dB} \text { scale })
$$

where the subscript denotes the clear sky conditions and $A_{i}(\mathrm{~dB})$ is the rain attenuation induced on a microwave link. We have outage when the received signal to noise ratio $S N R_{i}$ falls below a certain threshold $S N R_{t h}$, which depends on the transmission and receiver characteristics, as well as the desired QoS. In this case, when $S N R_{i} \leq S N R_{t h}$, we have :

$$
A_{i}=S N R_{c s}-S N R_{i} \geq S N R_{c s}-S N R_{t h}=F M_{i}
$$

where $F M_{i}$ is the fade margin of the link and corresponds to the value that the received signal under clear sky conditions can be attenuated without causing the link to fail.

\section{Diversity Reception with Signal Combining methods.}

\section{1) Joint Statistics.}

In the case of diversity reception, the signals received by the two HAPs can be combined in various ways, so as to achieve the optimum signal-to-noise ratio and consequently improve the system's outage probability. The total outage probability of the system depends on the joint statistics of the diversity links and on the method that is used to combine the diversity signals. The signal combining methods in use consist of the choice of the most powerful signal (SC: Selection Combining) and the linear combination of the signals received from the two diversity radiopaths by choosing appropriate weighting factors. The last method is further divided depending on the weighting factors selection. MRC (Maximal Ratio Combining) is used when the final signal's power is the sum of the diversity signals' powers, so the final SNR is the sum of the two SNRs.

2) Selection Combining (SC).

In the case of combining the received by the 2 HAPs signals by using the method Selection Combining (SC), the system's outage probability $P_{S C}$ is the probability of the event that the attenuations due to rain $A_{1}, A_{2}$ at the reception locations exceed simultaneously the two links' fade margins $F M_{1}, F M_{2}$. It can be calculated as previously presented, with the use of the joint PDF. The probability $P\left[A_{1} \geqslant F M_{1} A_{2} \geqslant F M_{2}\right]$ for the effective slant radiopaths of length $L_{s_{1}}, L_{s_{2}}$, according to Crane's simplification [7] is equal to the probability $P\left[A_{1} \geqslant F M_{1} \cos \phi_{1}, A_{2} \geqslant F M_{2} \cos \phi_{2}\right]$ for the projection paths of lengths $L_{d_{1}}, L_{d_{2}}$. Using mathematical manipulations that can be found in [4] and [5], the selection combining outage probability is given from the following final expression:

$$
P_{S C}=\frac{1}{2} \int_{u_{F M_{2}}}^{\infty} f_{U_{2}}\left(u_{2}\right) \cdot \operatorname{erfc}\left(\frac{u_{F M_{1}}-\rho_{n 12} u_{2}}{\sqrt{2\left(1-\rho_{n 12}^{2}\right)}}\right) d u_{2}
$$




$$
U_{i}=\frac{\ln \left(F M_{i} \cos \phi_{i}\right)-\ln A_{m_{i}}}{S_{a_{i}}},(i=1,2)
$$

In the above equations $\ln A_{m_{i}}, S_{a_{i}}$ are the the long-term rain attenuation statistical parameters of each link and can be found in terms of the statistical parameters of point rainfall rate that are given for every location in the world from ITU$\mathrm{R}$ rainmaps [8] and in terms of specific attenuation parameters [9] Finally $\rho_{n 12}$ is the logarithmic correlation coefficient. Its analytical expression can be found in [5].

3) Maximal Ratio Combining (MRC).

In the case of Maximal Ratio Combining (MRC) for the received signals of the 2 HAPs, the system's outage probability $P_{M R C}$ is the probability that the signal to noise ratio SNR of the final signal is below a certain threshold $S N R_{\text {th }}$ that assures reliable communication.

According to the Maximal Ratio Combining method (MRC) the signal-to-noise ratio under rain fades is equal in linear scale to the sum of the signal to noise ratio of the diversity signals, that is

$$
\left(\frac{S}{N}\right)_{M R C}=\left(\frac{S}{N}\right)_{1}+\left(\frac{S}{N}\right)_{2}
$$

which in logarothmic scale

$$
S N R_{M R C}=10 \log \left(10^{S N R_{1} / 10}+10^{S N R_{2} / 10}\right)
$$

or

$$
S N R_{M R C}=10 \log \left(10 \frac{S N R_{c s, 1}-A_{1}}{10}+10 \frac{S N R_{c s, 2}-A_{2}}{10}\right)
$$

If we apply Crane's simplification [7], if $A_{i}$ are the rain attenuations in the projection paths then the final SNR at $M R C$ receiver is given by:

$$
S N R_{M R C}=10 \log \left(10 \frac{S N R_{c s, 1}-A_{1} / \cos \phi_{1}}{10}+10 \frac{S N R_{c s, 2}-A_{2} / \cos \phi_{2}}{10}\right)
$$

The outage probability $P_{M R C}$ is the probability

$$
P_{M R C}=P\left(S N R_{M R C}<S N R_{t h}\right)
$$

, which can also be evaluated from the joint PDF of rain attenuation variables $f_{A_{1} A_{2}}\left(\alpha_{1}, \alpha_{2}\right)$, that means:

$$
P_{M R C}=\iint_{A} f_{A_{1} A_{2}}\left(\alpha_{1}, \alpha_{2}\right) d \alpha_{1} d \alpha_{2}
$$

whereas A the area for which the condition $S N R_{M R C}<S N R_{t h}$ holds. The area is defined by the curve $S N R_{M R C}=C\left(\alpha_{1}, \alpha_{2}\right)=S N R_{t h}$ and if $A_{2 \infty}$ and $\alpha_{1}\left(\alpha_{2}\right)$ are the roots of the equations $C\left(\infty, \alpha_{2}\right)=S N R_{t h}$ and $C\left(\alpha_{1}\left(\alpha_{2}\right), \alpha_{2}\right)=S N R_{t h}$ respectively then:

$$
P_{M R C}=\int_{A_{2 \infty} \alpha_{1}\left(\alpha_{2}\right)}^{\infty} \int_{A_{1} A_{2}}^{\infty}\left(\alpha_{1}, \alpha_{2}\right) d \alpha_{1} d \alpha_{2}
$$

In the above integral the transformation $U_{i}=\frac{\ln A_{i}-\ln A_{m_{i}}}{S_{a_{i}}},(i=1,2)$ can also be used so as to simplify the calculation to a simple integration.

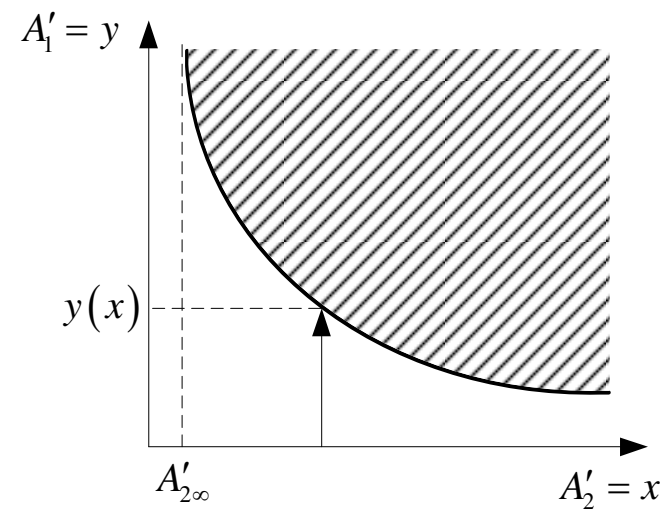

Figure 1 Region of integration in the $x-y$ plane for calculation of outage probability of dual-branch MRC.

The value $A_{2 \infty}$ which is the vertical asymptote in Fig. 2 can be easily calculated by a limiting process or algebraically.

In either case in order to evaluate the above probability in terms of the fade margins of the diversity links the transmission parameters have to be considered. The term fade margin $F M_{i}$ for the individual link $i$ hereby refers to the fade margin that the link would exhibit as if the MRC receiver was used for the link's reception with the absence of the other's branch signal. This way the analytical calculation proceeds as following: The fade margins are given by:

$$
F M_{i}=S N R_{c s, i}-S N R_{t h}
$$

and $S_{c s, i}$ is the received signal power at the link $i$ under clear sky conditions. From the link budget equation for the free space loss propagation

$$
S_{c s, i}=\frac{P_{t i} G_{t i} G_{r i} \lambda^{2}}{\left(4 \pi L_{i}\right)^{2}}
$$

where $P_{t i}$ the supply power, $G_{t i}$ the transmission antenna's gain, $G_{r i}$ the reception antenna's gain, $\lambda$ the wavelength and $L_{i}$ the radiopath's $i$ length (user - HAP i). Substituting for readability

$$
\frac{T_{1}}{T_{2}}=\frac{P_{t 1} G_{t 1} G_{r 1}}{P_{t 2} G_{t 2} G_{r 2}}
$$

as the ratio of the transmission specific parameters we obtain

$$
\frac{S_{c s, 1}}{S_{c s, 2}}=\frac{L_{2}^{2}}{L_{1}^{2}} \cdot \frac{T_{1}}{T_{2}}
$$




$$
\frac{\left(\frac{S}{N}\right)_{c s, 1}}{\left(\frac{S}{N}\right)_{c s, 2}}=\frac{L_{2}^{2}}{L_{1}^{2}} \cdot \frac{N_{2}}{N_{1}} \cdot \frac{T_{1}}{T_{2}}
$$

where $N_{1}, N_{2}$ the mean noise powers at HAPs 1,2 respectively. It is easily verified that:

$$
\frac{10^{F M_{1} 10}}{10^{F M_{2} / 10}}=\frac{\left(\frac{S}{N}\right)_{c s, 1}}{\left(\frac{S}{N}\right)_{c s, 2}}=\frac{L_{2}^{2}}{L_{1}^{2}} \cdot \frac{N_{2}}{N_{1}} \cdot \frac{T_{1}}{T_{2}}
$$

and

$$
\frac{\left(\frac{S}{N}\right)_{2}}{\left(\frac{S}{N}\right)_{1}}=\frac{\left(\frac{S}{N}\right)_{c s, 2} \cdot 10^{A_{1} / 10}}{\left(\frac{S}{N}\right)_{c s, 1} \cdot 10^{A_{2} / 10}}=\frac{L_{1}^{2}}{L_{2}^{2}} \cdot \frac{N_{1}}{N_{2}} \cdot \frac{T_{2}}{T_{1}} \cdot \frac{10^{A_{1} / 10}}{10^{A_{2} / 10}}
$$

By using the above relations and applying simple algebraic manipulations the outage condition for the MRC system is expressed

$$
10^{A_{1} / 10} \frac{L_{1}^{2}}{L_{2}^{2}} \cdot \frac{N_{1}}{N_{2}} \cdot \frac{T_{2}}{T_{1}}\left[\frac{1}{10^{F M_{2} / 10}}-\frac{1}{10^{A_{2} / 10}}\right]>1
$$

The above inequality in order to be true gives $A_{2}>F M_{2}$ (positive term in brackets). This way the outage probability is obtained by

$$
A_{1}>10 \log \left(\frac{L_{2}^{2} N_{2} T_{1}}{L_{1}^{2} N_{1} T_{2}} \cdot \frac{10^{A_{2} / 10} \cdot 10^{F M_{2} / 10}}{10^{A_{2} / 10}-10^{F M_{2} / 10}}\right)
$$

and

$$
A_{2}>F M_{2}
$$

If the rain attenuation values at the projection paths are used instead

$$
A_{1}>10 \log \left(\frac{L_{2}^{2} N_{2} T_{1}}{L_{1}^{2} N_{1} T_{2}} \cdot \frac{10^{A_{2} / 10 \cos \phi_{2}} \cdot 10^{F M_{2} / 10}}{10^{A_{2} / 10 \cos \phi_{2}}-10^{F M_{2} / 10}}\right) \cos \phi_{1}
$$

and

$$
A_{2}>F M_{2} \cos \phi_{2}
$$

Finally we have that the outage condition is equivalent to

$$
A_{2} \geq\left(S N R_{c s, 2}-S N R_{t h}\right) \cos \phi_{2}
$$

and

$$
\begin{aligned}
& A_{1}>S N R_{c s, 1} \cos \phi_{1}- \\
& -10 \log \left(10^{\frac{S N R_{t h}}{10}}-10^{\frac{S N R_{c s, 2}-A_{2} / \cos \phi_{2}}{10}}\right) \cos \phi_{1}
\end{aligned}
$$

The above calculated lower values for $A_{2}$ and $A_{1}$ correspond to the values $A_{2 \infty}$ and $\alpha_{1}\left(\alpha_{2}\right)$ previously mentioned. The outage probability can be calculated by means of the joint lognormal distribution with the use of the transformed R.V. $U_{1}, U_{2}$. In detail if we denote $\beta=\frac{N_{2} T_{1}}{N_{1} T_{2}}$ the ratio of the transmision and noise parameters (it can be assumed equal to unity) the probability $P_{M R C}$ is given by:

$$
\begin{gathered}
A_{2}>F M_{2} \cos \phi_{2} \Rightarrow \\
U_{2}>\frac{\ln \left(F M_{2} \cos \phi_{2}\right)-\ln A_{m 2}}{S_{a 2}}=u_{y_{1}} \\
A_{1}>10 \log \left(\frac{L_{2}^{2}}{L_{1}^{2}} \beta \cdot \frac{10^{A_{2} / 10 \cos \phi_{2}} \cdot 10^{F M_{2} / 10}}{10^{A_{2} / 10 \cos \phi_{2}}-10^{F M_{2} / 10}}\right) \cos \phi_{1} \Rightarrow \\
U_{1}>\frac{1}{S_{a 1}}\left[\ln (A)-\ln A_{m 1}\right]=g\left(u_{2}\right)
\end{gathered}
$$

where

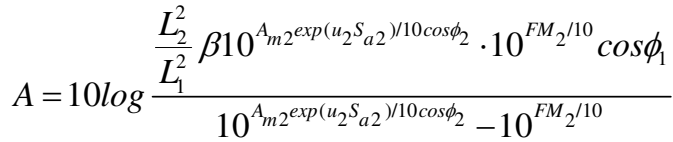

and, finally we have:

$$
\begin{gathered}
P_{M R C}=P\left[U_{1} \geqslant g\left(u_{2}\right), U_{2} \geqslant u_{y_{1}}\right]= \\
\frac{1}{2} \int_{u_{y_{1}}}^{\infty} f_{U_{2}}\left(u_{2}\right) \cdot \operatorname{erfc}\left(\frac{g\left(u_{2}\right)-\rho_{n} u_{2}}{\sqrt{2\left(1-\rho_{n}^{2}\right)}}\right) d u_{2}
\end{gathered}
$$

\section{NUMERICAL RESULTS AND DISCUSSION}

This section will present some numerical evaluations of the final analytical expressions. Table I summarizes the parameters that are used.

TABLE I.

DUAL-BRANCH DIVERSITY PARAMETERS

\begin{tabular}{|l|l|c|c|c|c|}
\hline \multirow{2}{*}{ Location } & \multicolumn{4}{|c|}{ Parameters } \\
\cline { 2 - 5 } & $\begin{array}{c}\text { Fading } \\
\text { Characteristics }\end{array}$ & $\begin{array}{c}\text { Long-term } \\
\text { Lognormal Rainfall } \\
\text { Rate Parameters }\end{array}$ & $\begin{array}{c}\text { System } \\
\text { Frequency }\end{array}$ & $\begin{array}{c}\text { Ground } \\
\text { distance } \\
\text { of HAPs }\end{array}$ \\
\hline $\begin{array}{l}\text { Athens, } \\
\text { Greece }\end{array}$ & $\begin{array}{l}\text { Joint } \\
\text { lognormal } \\
\text { fading distrib. }\end{array}$ & $R_{m}, S_{r}$ & $\begin{array}{c}0.0315, \\
1.6877\end{array}$ & $31 \mathrm{GHz}$ & $100 \mathrm{~km}$ \\
\hline
\end{tabular}

Figure 3 depicts the SC and MRC performance in terms of outage probability versus SNR threshold for a receiver located in Athens, Greece. The curves correspond to a ground receiver located in a direction vertical to the direction of the 2 HAPs at a distance of $100 \mathrm{~km}$. The curve for single branch (single HAP system) is also given for comparison. It can be seen that a MRC receiver outperforms the singlebranch receiver by around $5 \mathrm{~dB}$ for low threshold values and at about $3 \mathrm{~dB}$ for higher threshold values (low fade margins). When we compared to a SC receiver, the MRC technique has an advantage varying from $1 \mathrm{~dB}$ to $3 \mathrm{~dB}$ depending on the threshold. In links with low fade margin the efficiency of MRC is significantly better than that of SC, as the last one converges to that of the single branch. It can thus be concluded that, the MRC technique has a significant advantage over both SC and single-branch receivers.

Figure 4 investigates the impact of the distance between the 2 HAPs in the case of MRC. As it is expected, the greater 
the angular opening between the two branches the better the efficiency of the diversity scheme. This applies for SC as well. This factor should be considered in the decision of the spacing between HAPs in a HAPN, as well as any other parameters that relate to the selected architecture, such as coverage area and the minimum elevation angle at the user's terminal.

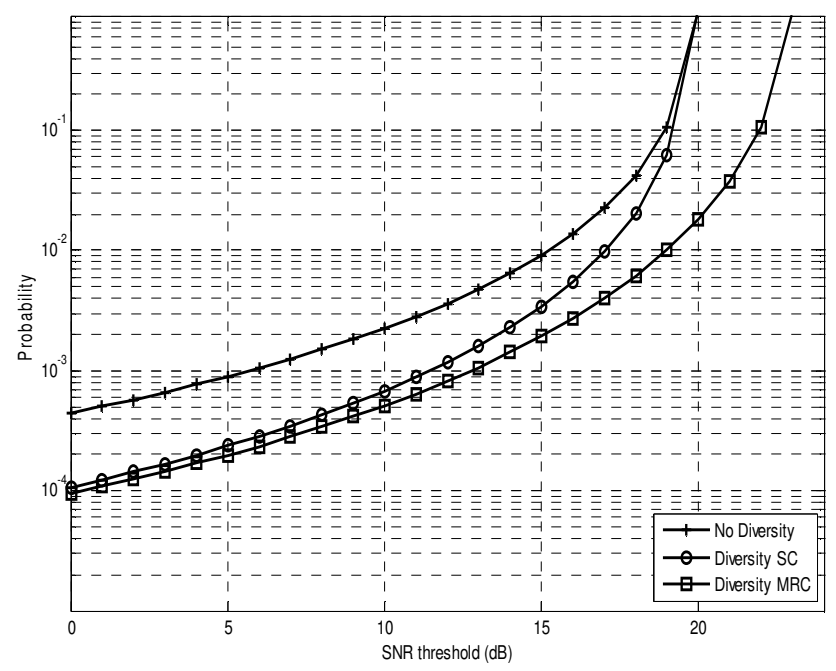

Fig. 3 MRC vs. $\mathrm{SC}$ and Single branch for $\mathrm{f}=31 \mathrm{GHz}, \omega$ : $90^{\circ}$ (direction vertical to HAPs connecting line) and ground distance $100 \mathrm{~km}$

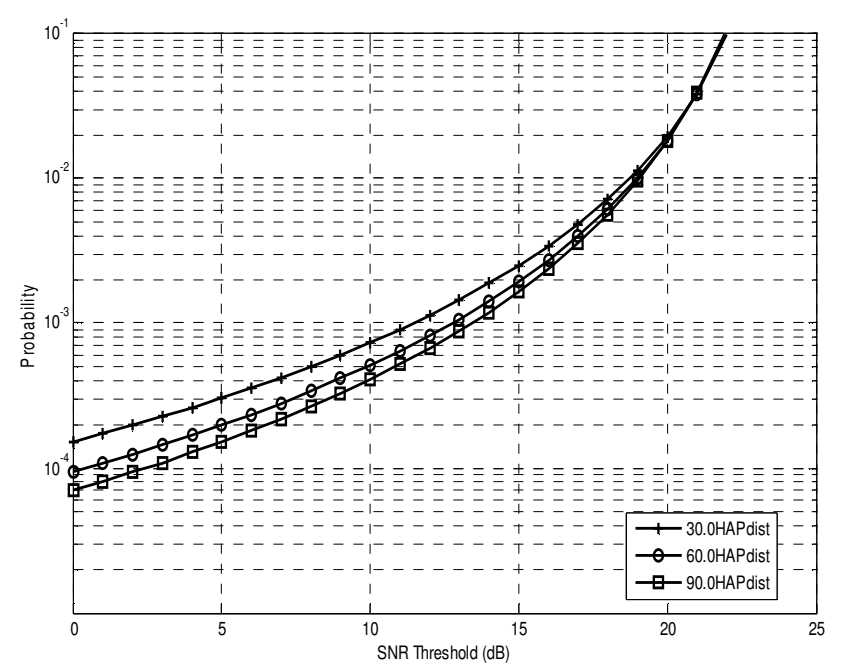

Fig. 4 MRC outage probabilities for HAP ground distances of 30, 60 and $90 \mathrm{~km}$

\section{CONCLUSION AND FUTURE WORK}

HAP Networks are a potential solution to several communication scenarios providing global connectivity. Techno-economic reasons make them more suitable for applying diversity schemes not only at site but also orbital. In this paper we have presented analytical models for the calculation MRC and SC outage probabilities. The double stratospheric system under consideration may be seen as a $2 \times 2$ MIMO system and important capacity calculations can be presented. This is a subject of future work. MRC superiority has been shown and the performance sensitivity in terms of the separation of the two HAPs has also been investigated.

\section{ACKNOWLEDGMENT}

This work has been supported in part by NTUA THALESMIMOSA Project funded by EU and Greece.

\section{REFERENCES}

[1] S. Karapantazis, F.-N.Pavlidou, "The Role of High Altitude Platforms (HAPs) in beyond 3G Networks", IEEE Wireless Communications, Vol 12, No 6, pp 33-41, Dec 2005.

[2] T. Tozera and D. Grace, "High-altitude platforms for wireless communications “, IET Electronics and Communications Engineering Journal, pp. 127-137, 2001.

[3] A. D. Panagopoulos, P. M. Arapoglou, P. G. Cottis, "Satellite Communications at $\mathrm{Ku}, \mathrm{Ka}$ and $\mathrm{V}$ bands, Propagation Impairments and Mitigation Techniques", IEEE Communication Surveys and Tutorials, $3^{\text {rd }}$ Quarter, pp.1-13, October 2004.

[4] A. D. Panagopoulos, E. M. Georgiadou, and John D. Kanellopoulos, "Selection Combining Site Diversity Performance in High Altitude Platform Networks", IEEE Communication Letters, vol. 11, no. 10, Oct. 2007.

[5] K. P. Liolis, A. D. Panagopoulos, and P. G. Cottis, "Multi-Satellite MIMO Communications at Ku-Band and Above: Investigations on Spatial Multiplexing for Capacity Improvement and Selection Diversity for Interference Mitigation", EURASIP Journal on Wireless Communications and Networking Volume 2007.

[6] ITU-R Rec. P.839-3, Rain height model for prediction methods, 2001.

[7] R.K. Crane, Electromagnetic Wave Propagation Through Rain, New York, Wiley, 1996.

[8] ITU-R Rec. P.837-5, "Characteristics of precipitation for propagation modeling," Geneva 2007.

[9] ITU-R Rec. P.838-3, "Specific attenuation model for rain for use in prediction method," Geneva 2005. 